\title{
Percutaneous endoscopic gastrostomy tube placement in amyotrophic lateral sclerosis: a case series with a multidisciplinary, team-based approach
}

\author{
Kelly Thomas a, Jason Schragerb, Robert Neelc, Loren Brook ${ }^{d}$ \\ University of Cincinnati Medical Center, Cincinnati, OH, USA
}

\section{Abstract}

\section{Introduction}

Amyotrophic lateral sclerosis (ALS) is a progressive, fatal neurodegenerative disease that leads to a progressive decline

Department of anternal Medicine (Kelly Thomas); 'burgery (Jason Schrager); 'Neurology (Robert Neel); ${ }^{\mathrm{d} G a s t r o e n t e r o l o g y ~(L o r e n ~ B r o o k), ~}$ University of Cincinnati Medical Center, Cincinnati, OH, USA

\section{Conflict of Interest: None}

Correspondence to: Kelly Thomas, University of Cincinnati, Department of Internal Medicine, Residency Office-MSB 6058, 231 Albert Sabin Way, Cincinnati, Ohio 45267-0557, USA,

e-mail: thoma2k4@ucmail.uc.edu

Received 9 March 2020; accepted 18 May 2020; published online 22 June 2020

DOI: https://doi.org/10.20524/aog.2020.0517 of motor functions at the spinal and bulbar level. In advanced disease, the main bulbar symptoms include dysphagia and dysarthria, which ultimately leads to malnutrition, weight loss, risk of aspiration, and increased morbidity and mortality. Other malnutrition risk factors in patients with ALS include anxiety, cognitive impairment, difficulty with self-feeding or meal preparation, and respiratory insufficiency. In ALS, malnutrition is common, with a prevalence ranging between $16 \%$ and $55 \%$ [1]. To maintain adequate oral intake, as well as an alternative route for medications, gastrostomy placement is recommended [2].

There are multiple methods of gastrostomy insertion, including surgical gastrostomy, percutaneous endoscopic gastrostomy (PEG), percutaneous radiologic gastrostomy (PRG), and per-oral image-guided gastrostomy (PIG). Surgical gastrostomy, whether open or through laparoscopy, is rarely used, as it has been shown to be associated with higher 
complication rates and greater mortality. Typically, it is reserved for patients who undergo abdominal surgery, or when other approaches are not possible [3]. Gastroenterologists insert PEG tubes with endoscopy using conscious sedation. Interventional radiologists perform PRG and PIG, performed under fluoroscopic guidance. PRG does not use conscious sedation, but only allows small-bore tube placement. PIG allows largebore tube insertion, yet requires minimal sedation and is a more complex procedure [3-5]. Although there are multiple methods of insertion, PEG tube placement is currently the standard procedure for enteral nutrition in ALS, as it is a relatively safe procedure with limited long-term morbidity and mortality that allows large-bore tube placement for feeding $[2,6]$.

Guidelines, based on consensus statements and expert opinion, have been developed to optimize the timing of gastrostomy tube placement on the basis of bulbar symptoms, nutritional status and respiratory function. The American Academy of Neurology recommends PEG insertion after the patient has lost more than $10 \%$ of their body weight and before their forced vital capacity (FVC) falls below 50\% [2,7]. The European Federation of Neurological Societies also recommends PEG insertion before a patient's FVC falls below $50 \%$ [8]. These guidelines take into consideration the patient's respiratory status, since patients with ALS are at increased risk for aspiration and postprocedural respiratory failure. However, there are insufficient data to support or refute any specific timing of PEG insertion [2,7].

Although there are some case studies evaluating the appropriate timing and procedural method of placement of gastrostomy tubes to reduce patient morbidity and mortality, no data have been published in relation to outcomes from a multidisciplinary, team-based approach to PEG tube placement. The University of Cincinnati Medical Center (UCMC) has developed a multidisciplinary approach to PEG placement. This incorporates neurology, anesthesiology, general surgery and gastroenterology into a patient-centered medical home with a focus on decreasing morbidity and mortality in ALS patients with various FVCs who have a need for PEG tube placement.

\section{Patients and methods}

This retrospective cohort study included ALS patients who had a PEG tube placed using a multidisciplinary approach at UCMC. Data were collected through password secured file and Provation endoscopy software.

\section{Patient characteristics}

Adult patients included were those with documented ALS via ICD-10 coding who received a PEG tube between August 2016 and August 2017. This date range allowed easily attainable data at the time when this multidisciplinary approach was first implemented. Study investigators from UCMC
Neurology and Gastroenterology departments decided on eligibility. Vulnerable populations were excluded, as well as any adult who did not receive a PEG from August 2016 to August 2017. Twenty-six patients met these study criteria.

The charts of these 26 patient were reviewed and data were collected regarding their preprocedural risks. These included age, presence of hyperlipidemia or coronary artery disease, hypertension, diabetes mellitus, chronic kidney disease, obstructive sleep apnea, FVC within 6 months of PEG insertion, and use of noninvasive ventilation prior to PEG (Table 1).

\section{Multidisciplinary approach being studied}

UCMC Neurology, Anesthesiology, General Surgery, and Gastroenterology departments were incorporated into a multidisciplinary approach to PEG placement. In this approach, once a patient was determined to be an appropriate candidate for PEG placement by neurology, the patient was referred to gastroenterology for a pre-PEG office visit to go over the procedural risks, benefits and expectations. The patient was then referred for a pre-anesthesia assessment with an anesthesiologist.

Table 1 Baseline characteristics of participants and their preprocedural risks

\begin{tabular}{ll}
\hline Characteristics & Value \\
\hline Age at PEG & $65.57 \pm 11.11$ \\
Placement (years) & Minimum 44 , maximum 86 \\
Sex & Male: $12(46 \%)$ \\
& Female: $14(54 \%)$ \\
Ethnicity & White: $23(88 \%)$ \\
& African American: $3(12 \%)$ \\
& Hispanic: $0(0 \%)$ \\
& Other: $0(0 \%)$ \\
Preprocedural & Hyperlipidemia/coronary artery disease \\
risks & Yes: $10(38 \%)$ \\
& No: $16(62 \%)$ \\
& Hypertension \\
Yes: $14(54 \%)$ \\
No: $12(46 \%)$ \\
Obstructive sleep apnea \\
Yes: $8(31 \%)$ \\
No: $18(69 \%)$ \\
Diabetes mellitus \\
Yes: $1(4 \%)$ \\
No: $25(96 \%)$ \\
Chronic kidney disease III or greater \\
Yes: 0 (0\%) \\
No: $26(100 \%)$ \\
Functional vital capacity within 1 year prior \\
to PEG \\
$54.38 \pm 13 \%$ \\
Minimum $34 \%$, maximum $87 \%$ \\
Noninvasive ventilation \\
Yes: $17(68 \%)$ \\
No: $8(32 \%)$ \\
\hline
\end{tabular}

Data are number (\%) or mean \pm standard deviation

PEG, percutaneous endoscopic gastrostomy 
The night before the procedure, the patient was admitted to the hospital under observation status. Neuromuscular respiratory parameters and lab work were checked to ensure there was no major change in pulmonary function or major concerns before the procedure. To optimize the patient's hydration status, intravenous fluids were provided. Anesthesia visited the patient to confirm that they would be safe for monitored anesthesia care, which allows for better control over sedation and a faster recovery. The next day, PEG placement was performed in the operating room with both gastroenterology and general surgery physicians. This procedure was performed in the operating room so that, if complications arose, the patient could more rapidly progress to a protected airway and/or a laparoscopic gastrostomy tube. To facilitate placement, minimize the amount and duration of anesthetic, and expedite the procedure, the same operative assistants-scrub techs and circulating nurses-were used, to ensure all were familiar with the exact progression of events. Special adjustments to the operative suite were made to minimize ambient light so that transillumination could be obtained more easily. An attending gastroenterologist performed the endoscopy and an attending general surgeon completed the abdominal portion of the procedure. Though these procedures were conducted at an academic center, no residents were utilized to expedite all facets of the procedure and again limit anesthesia timing.

Post-op, the patient was either returned to the neurology inpatient service or transferred to the neuroscience intensive care unit (NSICU). Inpatient nutrition would see the patient and prepare a nutritional discharge plan based on disease severity, increased caloric needs, and any other medical conditions. Once stable, the patient was discharged with close follow up. The patient's neurologist then monitored the patient's nutritional status at every follow-up visit and modified their nutrition plan as needed. In addition, the patient followed up with gastroenterology regarding appropriate tube care.

\section{Outcome measures}

The postprocedural outcomes obtained for each patient were immediate or delayed complications, hospital length of stay (LOS), admission to an intensive care setting, PEG usage at 6 and 12 months, readmissions for PEG-related complications at 6 and 12 months, and death at 3, 6, and 12 months. We then compared preprocedural risks and postprocedural outcomes with previously published studies on PEG placement in ALS patients under similar conditions, in order to analyze whether this multidisciplinary approach had better overall outcomes and should be considered a legitimate approach for placing gastrostomy tubes in all ALS patients in the future, even among those with an FVC $<50 \%$.

\section{Statistical analysis}

Univariate analysis of the data was done using analysis of variance (ANOVA) for continuous variables and chi-square test for categorical variables. Logistic regression was used to further analyze the variables found to be significant on univariate analysis and the variables determined to be confounding and effect modifiers.

\section{Results}

Patients had a mean age of 66 years at PEG placement; $54 \%$ were female and $88 \%$ were Caucasian. The mean FVC prior to PEG was 54\%; 9 patients had an FVC less than $50 \%$ and 18 patients were on noninvasive ventilation prior to their procedure. All patients were involved in UCMC's multidisciplinary, team-based approach; thus, every patient had encounters with neurology, gastroenterology, and anesthesiology prior to PEG placement. All procedures were performed in the operating room with a gastroenterologist and surgeon present; the success rate was $100 \%$, as there were no surgical or anesthesia concerns preventing PEG tube placement.

Regarding complications, there were 2 minor complications: hypotension self-resolved on recheck and pain at PEG site. There were no major complications. All patients were monitored after PEG placement inpatient. Only 2 patients required NSICU status after PEG placement, while the others were monitored on medicine ward floors. The mean LOS was 3 days, the minimum being 2 days and the maximum 4 days.

On follow up, there were no PEG-related complications or death. At 30 days there was one death, making the 30-day mortality $4 \%$. Mortality at 3, 6, and 12 months was $12 \%, 22 \%$, and $30 \%$ respectively (Table 2 ). All patients alive at 3, 6, and 12 months were using their PEG tubes without issues.

\section{Discussion}

The UCMC multidisciplinary approach had fewer minor and major complications when compared to other studies. UCMC only had 2 minor complications: mild hypotension and pain at the PEG site. Only a few studies have been published that describe the minor and major complications associated with PEG tube insertion in similar patient populations (Table 3). One was a retrospective study of 57 patients, in which 4 patients had major complications of aspiration and respiratory failure and 4 patients had minor complications of local pain, minor bleeding, tube dislodgement and unsuccessful placement [6].

Table 2 Mortality of patients who received a percutaneous endoscopic gastrostomy tube using The University of Cincinnati Medical Center's multidisciplinary approach

\begin{tabular}{lcc}
\hline Mortality & Yes & No \\
\hline Death at 3 months & $3(12 \%)$ & $18(88 \%)$ \\
Death at 6 months & $5(22 \%)$ & $18(78 \%)$ \\
Death at 12 months & $7(30 \%)$ & $16(70 \%)$ \\
\hline Data are number of patients (percentage)
\end{tabular}


Table 3 Comparison of The University of Cincinnati Medical Center (UCMC)'s multidisciplinary approach to other studies

\begin{tabular}{lccccccc}
\hline Study & $\begin{array}{c}\text { Number of } \\
\text { patients }\end{array}$ & Age & Sex & Mean FVC & $\begin{array}{c}\text { Failed PEG-tube } \\
\text { placement rate }\end{array}$ & $\begin{array}{c}\text { Major } \\
\text { complications }\end{array}$ & $\begin{array}{c}\text { Minor } \\
\text { complications }\end{array}$ \\
\hline $\begin{array}{l}\text { UCMC's } \\
\text { Approach }\end{array}$ & 26 & $65.57 \pm 11.11$ & $\begin{array}{l}46 \% \text { Male, 54\% } \\
\text { Female }\end{array}$ & $54.38 \pm 13$ & $0(0 \%)$ & $0(0 \%)$ & $2(7.7 \%)$ \\
$\begin{array}{l}\text { Allen, et al [9] } \\
2013\end{array}$ & 57 & $61.7 \pm 11.3$ & $\begin{array}{l}61.4 \% \text { Male, } \\
38.6 \% \text { Female }\end{array}$ & $54.4 \pm 18.2$ & $9(15.7 \%)$ & $4(7 \%)$ & $4(7 \%)$ \\
$\begin{array}{l}\text { Russ, et al [6] } \\
2015\end{array}$ & 21 & 58.9 & not stated & 50.20 & $1(4.7 \%)$ & $2(9.4 \%)$ & $7(33.1 \%)$ \\
$\begin{array}{l}\text { Dorst, et al [10] } \\
2017\end{array}$ & 89 & $63.1 \pm 10$ & $48.3 \%$ Male & $51.4 \pm 21.4$ & not stated & $1(1.1 \%)$ & $37(41.57 \%)$ \\
\hline
\end{tabular}

Data are number, number \pm standard deviation or number (\%)

FVC, forced vital capacity; PEG, percutaneous endoscopic gastrostomy

The second study was a retrospective study of 21 patients, in which 2 patients had major complications, defined as systemic and life threatening, and 7 patients had minor complications, described as local and non-life-threatening [9]. The third study was a prospective observational study of 89 patients, in which 1 patient had a major complication of an abscess and 37 patients had minor complications of dislocation, problems with handling, local bleeding, peritonitis, local inflammation and local pain [10]. These studies all included patients who had ALS with various FVCs, with the 3 FVC means ranging from 50-55, similar to our patient population. In addition, UCMC's multidisciplinary approach had no failed PEG-tube placements, while all other studies reviewed regarding PEG placement in patients with ALS had at least 1 failed PEG-tube placement with similar population sizes (Table 3).

The UCMC multidisciplinary approach had similar LOS compared to similar published data. A retrospective review of patients with neurodegenerative disease showed that $40 \%$ patients exceeded 3 days of hospitalization after PEG tube placement, similar to the $38 \%$ who exceeded 3 days of hospitalization at UCMC [11]. Although lowering the complication rate was initially expected to help decrease hospital LOS, this study did not show this effect. However, data were limited on LOS regarding patients with ALS post PEG placement, as only 1 published study documented LOS for comparison.

In our study, there was 1 death at 30 days. Upon further investigation, this patient underwent expedited multidisciplinary evaluation and insertion of PEG while an inpatient, because of concern about an aggressive form of ALS requiring immediate attention. With this multidisciplinary approach, she survived PEG surgery but died 29 days later. It is difficult to determine whether her death after surgery was due to postoperative complication(s) or terminal progression of her aggressive form of ALS.

Overall, UCMC's multidisciplinary approach had $4 \%$ mortality at 30 days. A meta-analysis of 17 published studies determined that the weighted estimate of 30-day mortality following PEG tube insertion was 10\% (95\% confidence interval [CI] 5-15\%) and the weighted estimate of 30-day mortality following PRG/PIG insertion was 6\% (95\%CI 3-9\%) [3]. Thus, the 30-day mortality rate for both PEG and RIG/PIG insertion using UCMC's multidisciplinary approach to PEG placement is lower than that seen in the literature for both PEG and RIG/PIG. In addition, 3 studies that compared mortality in patients who received PEG and RIG/PIG showed that both of these methods had a median survival of less than 1 year, while UCMC's study had $70 \%$ surviving after 1 year [12]. Although this study was limited by the fact that data collection occurred solely in 2017, it still shows the benefits of using a multidisciplinary approach when compared to the other case studies previously published. These data are from the time UCMC's multidisciplinary approach was first implemented and thus show the benefits of this approach, even prior to any system improvements. UCMC continues to use a multidisciplinary approach today for PEG placement in ALS patients, given the benefits this approach has shown in the past.

In conclusion, ALS is a disorder of adults characterized by a decline in motor function that mainly affects muscles at the spinal and bulbar level. Regardless of the technique of insertion, the goal of gastrostomy placement is to increase enteral nutrition and ultimately prolong survival in ALS. UCMC has developed a multidisciplinary approach to PEG placement. This approach incorporates neurology, anesthesiology, general surgery and gastroenterology into a patient-centered medical home with a focus on decreasing morbidity and mortality in ALS patients. We have not found any previously described cases or studies that used this approach and, when our findings are compared to previously published outcome data, UCMC's approach had fewer complications, higher insertion success rates, and comparable LOS. These data support the effectiveness of a multidisciplinary approach to increase the success and survival rates of patients with ALS, even if they have respiratory compromise (FVC $<50 \%$ ) at the time of PEG insertion.

\section{Acknowledgments}

We thank the patients who consented to participate in UCMC's team-based approach to PEG placement. We also thank Jason Blackard, $\mathrm{PhD}$ for aiding in the revision of this paper. 


\section{Summary Box}

\section{What is already known:}

- Malnutrition in patients with amyotrophic lateral sclerosis (ALS) is common, with a prevalence ranging between $16 \%$ and $55 \%$

- In ALS patients at high risk for malnutrition, percutaneous endoscopic gastrostomy (PEG) tube placement is recommended to maintain nutrition and provide an alternative route for medications

- American Academy of Neurology and European Federation of Neurological Societies guidelines recommend PEG insertion before a patient's forced vital capacity (FVC) falls below 50\%, based on previously published studies

\section{What the new findings are:}

- The University of Cincinnati Medical Center (UCMC) has implemented a multidisciplinary approach to PEG placement with the goal of decreasing morbidity and mortality in those with ALS

- UCMC's multidisciplinary approach to PEG placement had less complications, higher insertion success rates and comparable hospital length of stay compared to previously published studies of PEG insertion in ALS patients

- This multidisciplinary approach challenges current guidelines, as the data support greater success and survival rates among ALS patients who undergo PEG placement, even those who have an FVC $<50 \%$ at the time of PEG insertion

\section{References}

1. Genton L, Viatte V, Janssens JP, Héritier AC, Pichard C. Nutritional state, energy intakes and energy expenditure of amyotrophic lateral sclerosis (ALS) patients. Clin Nutr 2011;30:553-559.

2. Sarfaty M, Nefussy B, Gross D, Shapira Y, Vaisman N, Drory V. Outcome of percutaneous endoscopic gastrostomy insertion in patients with amyotrophic lateral sclerosis in relation to respiratory dysfunction. Amyotroph Lateral Scler Frontotemporal Degener 2013; 14:528-532.

3. Stavroulakis T, Walsh T, Shaw PJ, McDermott CJ. Gastrostomy use in motor neurone disease (MND): a review, meta-analysis and survey of current practice. Amyotroph Lateral Scler Frontotemporal Degener 2013;14:96-104.

4. Perona F, Castellazzi G, De Iuliis A, Rizzo L. Percutaneous radiologic gastrostomy: a 12-year series. Gut Liver 2010;4 Suppl 1:S44-S49.

5. Rio A, Ampong MA, Turner MR, et al. Comparison of two percutaneous radiological gastrostomy tubes in the nutritional management of ALS patients. Amyotroph Lateral Sclerosis 2005;6:177-181.

6. Russ KB, Phillips MC, Wilcox CM, Peter S. Percutaneous endoscopic gastrostomy in amyotrophic lateral sclerosis. Am J Med Sci 2015;350:95-97.

7. Miller RG, Jackson CE, Kasarskis EJ, et al; Quality Standards Subcommittee of the American Academy of Neurology. Practice parameter update: the care of the patient with amyotrophic lateral sclerosis: drug, nutritional, and respiratory therapies (an evidence-based review): report of the Quality Standards Subcommittee of the American Academy of Neurology. Neurology 2009;73:1218-1226.

8. Andersen PM, Abrahams S, Borasio GD, et al; EFNS Task Force on Diagnosis and Management of Amyotrophic Lateral Sclerosis:. EFNS guidelines on the clinical management of amyotrophic lateral sclerosis (MALS) - revised report of an EFNS task force. Eur J Neurol 2012;19:360-375.

9. Allen JA, Chen R, Ajroud-Driss S, et al. Gastrostomy tube placement by endoscopy versus radiologic methods in patients with ALS: a retrospective study of complications and outcome. Amyotroph Lateral Scler Frontotemporal Degener 2013;14:308-314.

10. Dorst J, Dupuis L, Petri S, et al. Percutaneous endoscopic gastrostomy in amyotrophic lateral sclerosis: a prospective observational study. J Neurol 2015;262:849-858.

11. Sarkar P, Cole A, Scolding NJ, Rice CM. Percutaneous endoscopic gastrostomy tube insertion in neurodegenerative disease: a retrospective study and literature review. Clin Endosc 2017;50:270-278.

12. Shaw AS, Ampong MA, Rio A, et al. Survival of patients with ALS following institution of enteral feeding is related to pre-procedure oximetry: a retrospective review of 98 patients in a single centre. Amyotroph Lateral Scler 2006;7:16-21. 